\title{
Heat Transfer in an Enhanced Cable Insulation Scheme for the Superconducting Magnets of the LHC Luminosity Upgrade
}

\author{
Pier Paolo Granieri, Paolo Fessia, David Richter, and Davide Tommasini
}

\begin{abstract}
The next generation of superconducting magnets for the interaction regions of particle colliders, as well as for fast cycled accelerators, will be confronted with large heat loads. In order to improve the evacuation of heat from the $\mathrm{Nb}$ - $\mathrm{Ti}$ coil towards $\mathrm{He}-\mathrm{II}$ bath, a porous (enhanced) all-polyimide cable insulation scheme was proposed recently. The first results were promising, featuring a larger permeability to helium with respect to existing schemes under low compressive stress. In this paper we present an extended experimental study of heat transfer through the Enhanced Insulation into He-II bath, and comparison to the standard LHC insulation, at different levels of applied pressure. The thermal coupling between adjacent cables was investigated, as well as the impact of a localized heat deposition versus a distributed one. The results of this study show that, up to high pressure levels, the enhanced insulation scheme can provide a major improvement of heat transfer compared to the standard scheme used in the main LHC magnets.
\end{abstract}

Index Terms-Accelerator superconducting magnets, enhanced heat transfer, LHC upgrade phase I, porous cable insulation.

\section{INTRODUCTION}

$\mathbf{S}$ UPERCONDUCTING magnets require that every turn of the coils is dielectrically insulated from adjacent turns, as well as from other metallic components of the magnet. Due to the large energy stored in the magnetic field, the choice of an appropriate cable electrical insulation is fundamental to ensure the safety of the device against electrical short circuit.

The cable electrical insulation affects the magnet thermal behavior, because it forms the main thermal resistance between conductor and coolant. It determines the cooling of the coil, which is essential in maintaining the superconducting state of the conductors against heat generation or deposition.

Heat can be deposited in the magnet due for instance to beam loss, to the ramping of magnetic field, or due to the debris of collisions in magnets close to the interaction regions. This last item is especially important in view of the LHC upgrade phase

\footnotetext{
Manuscript received October 18, 2009. First published March 01, 2010; current version published May 28, 2010. This project has received funding from the European Community's Seventh Framework Programme (FP7/2007-2013) under the Grant Agreement 212114.

P. P. Granieri is with CERN, Technology Department, Geneva, Switzerland, and also with Ecole Polytechnique Federale of Lausanne (EPFL), Lausanne, Switzerland (e-mail: pier.paolo.granieri@cern.ch).

P. Fessia, D. Richter, and D. Tommasini are with CERN, Technology Department, Geneva, Switzerland (e-mail: pier.paolo.granieri@cern.ch).

Color versions of one or more of the figures in this paper are available online at http://ieeexplore.ieee.org.

Digital Object Identifier 10.1109/TASC.2010.2040377
}
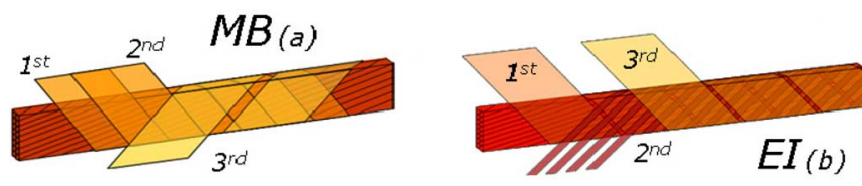

Fig. 1. Scheme of the LHC main dipole (MB) insulation (a), and of the Enhanced Insulation (EI) (b).

I [1], requiring replacement of the existing low- $\beta$ triplets in the CMS and Atlas experimental areas with more powerful ones [2]. The new quadrupole MQXC will be exposed to increased heat deposition [3], calling therefore for a stronger resistance to heat loads.

A new concept of helium-permeable cable insulation, hereafter referred to as Enhanced Insulation, was proposed in the last years to improve the coil thermal performance by better exploiting the cooling potential of He-II, while preserving electrical protection. The first investigations showed an increased porosity to air with respect to the LHC standard insulation [4], and an increased heat extraction in $1.9 \mathrm{~K}$ bath under low compressive stress [5].

Later, a systematic test campaign was launched to fully qualify the proposed Enhanced Insulation. This paper reports on the results of heat transfer measurements performed in He-II, aiming at verifying the enhanced thermal properties under the mechanical stress typical for magnet operation. The electrical and mechanical performance was also assessed to be satisfactory, as described in a separate paper [6].

\section{ENHANCED CABLE INSULATION}

The standard insulation of the LHC main dipoles (MB) is composed of 3 wrappings of polyimide tapes [7], as shown in Fig. 1(a), consisting of:

- The $1 \mathrm{st}$ and 2 nd tape $11 \mathrm{~mm}$ wide and $50 \mu \mathrm{m}$ thick. The 2 nd one is $50 \%$ overlapped with the 1 st one;

- The 3rd tape $9 \mathrm{~mm}$ wide and $69 \mu \mathrm{m}$ thick with, on the outer face, a thin coat of polyimide adhesive activated by thermal treatment.

The first two tapes ensure the electrical insulation by minimizing the risk of punch-through and providing a sufficiently long surface path for electrical discharge. The 3rd layer is intended to protect them and to provide cohesion between coil turns. It is wrapped with a spacing of $2 \mathrm{~mm}$ to make the coil 
porous to He-II, thus allowing larger heat evacuation with respect to sealed insulation schemes [8].

The transfer of heat from cable to He-II bath can be indeed schematized into two different components: one through the micro-channels between the insulation tapes, the other by solid conduction across the insulation bulk [9].

The proposed enhanced cable insulation scheme (EI) aims at improving the heat exchange of the $\mathrm{Nb}-\mathrm{Ti}$ coils with the He-II bath through these micro-channels. The layout, shown in Fig. 1(b), was slightly modified from [4] and [5] to enable the wrapping of commercially available tapes in a semi-industrial environment. It is hereafter named EI\#4, which is the version retained among several new insulation schemes. It consists of:

- The 1st tape $9 \mathrm{~mm}$ wide and $50 \mu \mathrm{m}$ thick;

- The 2nd tape $3 \mathrm{~mm}$ wide and $75 \mu \mathrm{m}$ thick, cross-wrapped with the others;

— The 3rd tape $9 \mathrm{~mm}$ wide and $69 \mu \mathrm{m}$ thick, with the same polyimide adhesive as in the LHC MB insulation, 50\% overlapped with the 1st tape.

The three polyimide layers are wound with spacing: $1 \mathrm{~mm}$ for the $1 \mathrm{st}$ and $3 \mathrm{rd}$ tape, $1.5 \mathrm{~mm}$ for the $2 \mathrm{nd}$ one. The electrical insulation is provided by the combined effect of the 1 st and 3rd tape, whereas the role of the 2nd one is to avoid a direct overlap between them, thus providing at the same time for cooling channels and dielectric insulation. As for the LHC MB insulation, the 3rd tape also provides mechanical stability.

\section{Heat Transfer Measurements}

The thermal characterization of the Enhanced Insulation with respect to the LHC standard one was performed on cables stacks representative of a coil, as in [5]. The results are consistent with those obtained in [10] where a segment of a LHC production coil was measured, thus validating the consistency of this experimental method.

\section{A. Sample Preparation and Experimental Setup}

Two test samples were prepared from a resistive $\mathrm{CuNi}_{10} \mathrm{wt} \%$ cable with the same geometry as the LHC Cable 01 [7], and insulated according to the two schemes described in Section II. Six cables were alternately stacked to compensate for the cable keystone, thus forming a rectangular stack. The stack was cured according to a bonding cycle consisting of a pressure cycle up to $130 \mathrm{MPa}$ at a temperature of $190^{\circ} \mathrm{C}$. It is worth noting that this choice is conservative, because the curing pressure of the LHC main superconducting magnets is $80 \mathrm{MPa}$.

Five adjacent cables were connected at their extremities to a current supply, the 6th one being necessary only for geometrical reasons. The cables were uniformly heated by supplying current to the resistive strands, as in [11]. The heat generated was calculated, with an error smaller than $0.1 \%$, using the measurement of the voltage at the cable extremities and of the injected current.

The central of the five cables was instrumented with nine $\mathrm{AuFe}_{0.07 \mathrm{at} \%}$-Chromel thermocouple junctions (TCJs). In order to avoid short-circuits under the high compression loads acting on the sample, grooves were machined in the strands before insulating the cable, to host the TCJs and their wires. After the

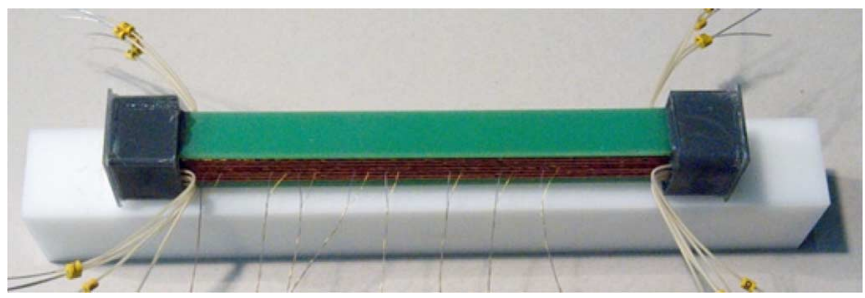

Fig. 2. Instrumented sample, ready to be installed in the sample-holder.

curing cycle of the sample, the 3rd insulation tape of the cable adjacent to the one to instrument was cut along the cable's small side. This cable was temporarily taken out from the stack, while its 1st and 2nd tape remained intact. Small squares of insulation were removed from the central cable's flat side at the end of each groove, allowing the varnish insulated TCJs to be installed. The TCJs wires were drawn along the groove towards the thick edge of the cable and through the insulation outwards. The TCJs were then sealed using epoxy resin.

The relative error of the measured temperature difference between the cable and the He-II bath was evaluated to be less than $3 \mathrm{mK}$ or $3 \%$, whichever is bigger.

The sample preparation was completed by adding $3 \mathrm{~mm}$ thick fiberglass plates on each side of the stack, both for thermal and mechanical reasons, and epoxy resin plugs on its extremities to prevent longitudinal parasitic cooling (Fig. 2).

The sample was then installed in an Al-alloy spring-like holder, allowing to apply a compressive pressure on the cables flat side over the central $150 \mathrm{~mm}$. Both small sides of the cables are in direct contact with the He-II bath over the central $156 \mathrm{~mm}$. Finally, the sample-holder was installed in a Claudet-bath cryostat.

The $0.1 \mathrm{MPa}$ pressurized bath was regulated to the temperature of $1.9 \mathrm{~K}$, stabilized within $0.2 \mathrm{mK}$, and measured using three Ge temperature sensors with absolute errors less than $10 \mathrm{mK}$.

\section{B. Heating Configurations}

The experimental setup allows measuring the sample in different heating configurations:

Symmetric heating, i.e. heating up of one, three or five central conductors, allowing studying the impact of a localized heat deposition versus a distributed one.

Asymmetric heating, such as the case where the heated cable is adjacent to the central, instrumented one, to measure the thermal coupling between the cables.

\section{Assessment of the Applied Pressure}

The actual pressure applied on the sample at cryogenic temperature was verified through a dedicated measurement. An Al-alloy bar was instrumented with strain gauges and placed under the sample. The sample holder was closed at defined pressure and cooled to $77 \mathrm{~K}$. The measurements showed no pressure reduction up to levels of $25 \mathrm{MPa}$, whereas an increase was observed for higher pressure levels $(10 \%$ at $75 \mathrm{MPa})$. Values indicated in Section IV refer to the pressure applied at room temperature. 


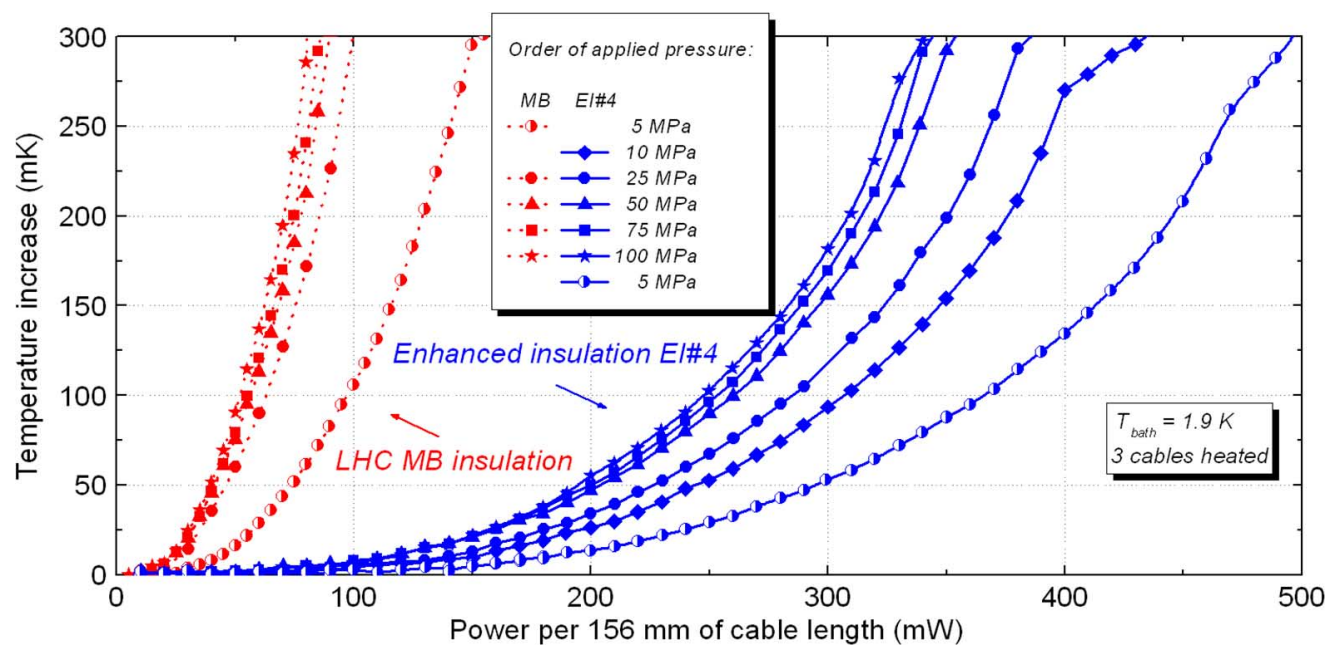

Fig. 3. Temperature increase as a function of the power dissipated in the central conductor, in a He bath at $1.9 \mathrm{~K}$. The samples insulated with the MB and EI\#4 insulation schemes were tested at several applied pressure levels. The lines are a guide to the eye.

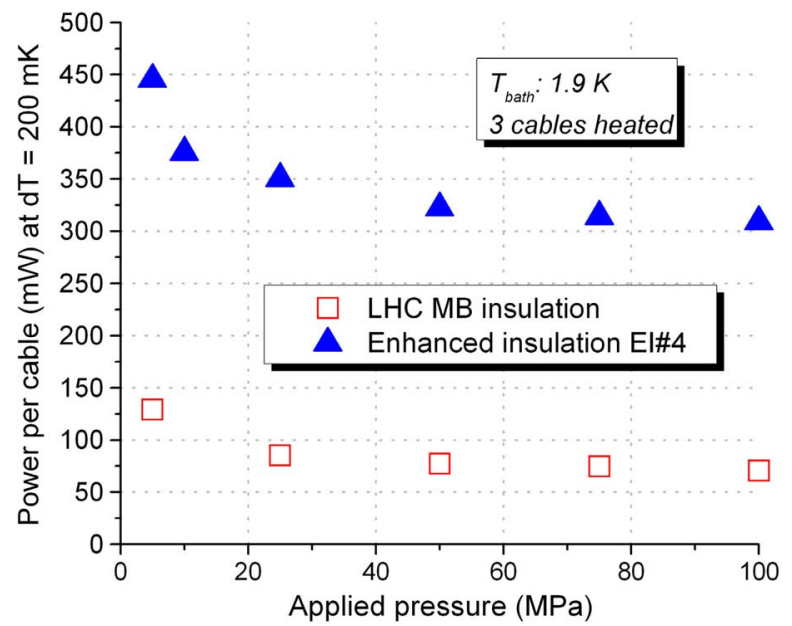

Fig. 4. Power dissipated in the MB and EI\#4 samples as a function of the applied pressure, for a constant temperature increase of $200 \mathrm{mK}$.

\section{RESULTS AND ANALYSIS}

\section{A. Heat Transfer Results}

The measurements aim at correlating the cable steady-state temperature increase $\Delta \mathrm{T}_{\mathrm{c}}$ to the dissipated power in the cable. Fig. 4 reports the results obtained for the two samples described in Section III up to a $\Delta \mathrm{T}_{\mathrm{c}}$ of $300 \mathrm{mK}$, for several applied pressure levels. The power indicated on the $\mathrm{x}$-axis refers to the central cable, while three central cables were heated up simultaneously.

The enhanced cable insulation allows evacuating about 4.5-times more power than the LHC standard one at any pressure.

The higher the applied pressure is, the lower the heat transfer is, as expected. This effect is more evident at low pressures, when the cable is softer [12], hence the micro-channels undergo a large size reduction. This feature is clearly shown in Fig. 5, reporting the power extraction as a function of the pressure, for

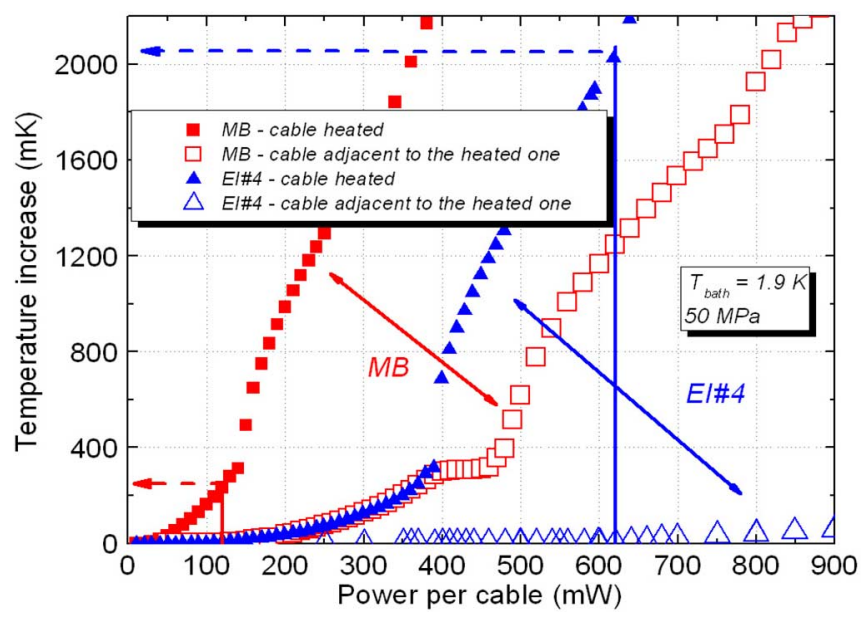

Fig. 5. Temperature increase of the heated cable and of the adjacent one as a function of the power dissipated in the heated cable. The MB and EI\#4 samples are considered, at a pressure of $50 \mathrm{MPa}$.

a constant $\Delta \mathrm{T}_{\mathrm{C}}$ of $200 \mathrm{mK}$, which tends to an asymptotic value for high pressures.

Despite the reduction of the heat transfer that can be observed on both samples with increasing the pressure, the Enhanced Insulation provides at least four times better power extraction than the LHC standard one also at the highest pressure levels. This means that the component of heat transfer through the micro-channels is still efficient.

The measurement at $100 \mathrm{MPa}$ on the EI\#4 sample was repeated after 20 days, keeping in the meantime the sample under a $100 \mathrm{MPa}$ pressure. The difference with the previous measurement was not significant, meaning that the creep effect in this time scale has no impact on heat transfer. This is probably due to the fact that the creep effect is more evident during the first 3-4 days [6], which is the time the sample usually remains under pressure before being measured.

The change of heat transfer regime occurs at a $\Delta \mathrm{T}_{\mathrm{c}}^{*}$ slightly above $300 \mathrm{mK}$ for the MB sample and slightly below for the EI\#4 one. This may be related to the He $\lambda$ transition and to 


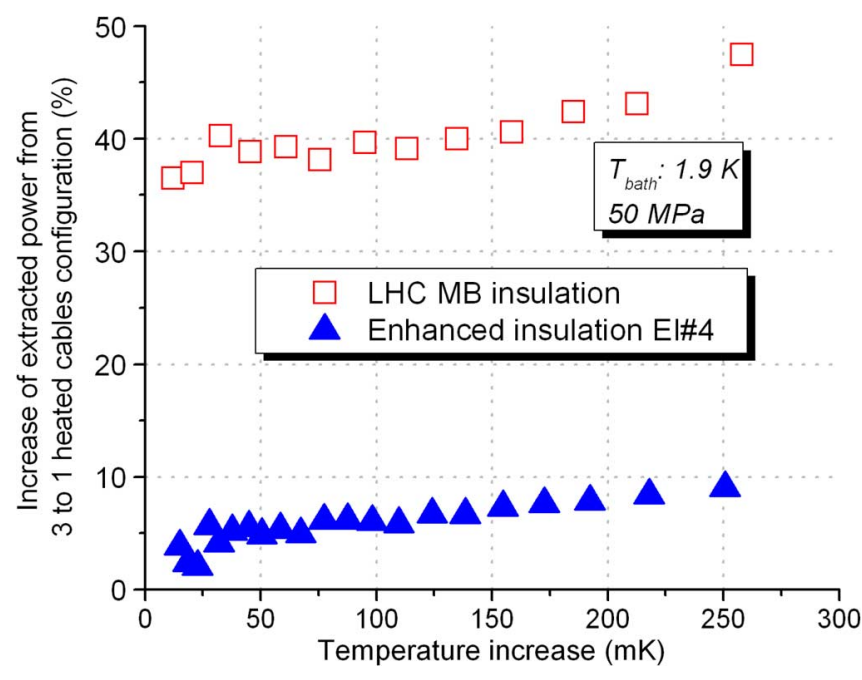

Fig. 6. Increase of extracted power as a function of the temperature increase, passing from 3 to 1 heated cables configuration. The MB and EI\#4 samples are considered, at a pressure of $50 \mathrm{MPa}$.

the status of saturation in the micro-channels, and requires more investigations.

\section{B. Thermal Coupling Between Cables}

The measurement of the heating configurations with the central cable heated, and of the asymmetric one described in Section III-B provided information on the thermal coupling between adjacent cables. As it can be seen in Fig. 6, the $\Delta T_{c}$ of the heated cable at which the adjacent (not heated) cable starts to increase its temperature is around $235 \mathrm{mK}$ for the MB sample, and around $2100 \mathrm{mK}$ for the EI\#4 one. Hence the Enhanced Insulation allows adjacent cables to be more thermally decoupled than the MB insulation does, thanks to an efficient heat transfer between the cable and the bath.

\section{Localized vs. Distributed Heat Deposition}

Measurements carried out in symmetric configuration with one cable heated (representing a localized heat deposition) showed a more efficient heat extraction than for three or five cables heated (distributed heat deposition). Fig. 7 shows the increase in terms of extracted power, if passing from three to one heated cable configuration. In the case of the MB sample, the central cable can transfer more heat to the adjacent cables than in the EI\#4 sample. This is a confirmation of the stronger thermal coupling of adjacent cables insulated with the MB scheme. The EI\#4 scheme, on the other hand, allows a better access of He-II to the strands through the micro-channels network, thus explaining the weaker thermal coupling.

\section{Ongoing Work}

The measurement of another Enhanced Insulation scheme is planned, to assess the impact of the micro-channels size.

Though the used "stack method" is an irreplaceable and representative way to study the coil's thermal behavior and to compare different cable insulation schemes, it does not give informa- tion about the heat flux through each cable face. This is needed to obtain knowledge of the coil cross-section thermal behavior, to provide a phenomenological explanation of the EI improved heat transfer, and to model the heat transport through the microchannels, which topology depends on the face and on the applied pressure. Experiments are planned to fill this lack.

\section{CONCLUSION}

A new all-polyimide insulation scheme was proposed to improve the cooling of superconducting coils, allowing He-II to better permeate through its structure while maintaining satisfactory electrical performances. The heat transfer through the proposed Enhanced Insulation was measured in He-II, showing an increased heat transfer capacity by at least a factor of four with respect to the LHC standard insulation scheme. It was proved that this improvement does not reduce with the applied pressure, up to the levels typical of magnet operation. The proposed Enhanced Insulation is therefore a suitable candidate for the low- $\beta$ superconducting quadrupoles for the LHC upgrade phase-I.

\section{ACKNOWLEDGMENT}

The authors would like to thank S. Luziex for insulating and curing the samples and M. Guinchard and F. Regis for participating in the pressure measurements, as well as L. Bottura, L.-R. Oberli, and L. Rossi for their support.

\section{REFERENCES}

[1] J. P. Koutchouk, L. Rossi, and E. Todesco, A Solution for Phase-One Upgrade of the LHC Low-Beta Quadrupoles Based on Nb-Ti CERN, Geneva, Switzerland, LHC Project Report 1000, 2007.

[2] P. Fessia and P. P. Granieri et al., "A $120 \mathrm{~mm}$ bore quadrupole for the phase I LHC upgrade," IEEE Trans. Appl. Supercond., submitted for publication.

[3] R. Ostojic et al., Conceptual Design of the LHC Interaction Region Upgrade: Phase-I CERN, Geneva, Switzerland, LHC Project Report 1163, 2008.

[4] M. La China and D. Tommasini, "Comparative study of heat transfer from $\mathrm{Nb}-\mathrm{Ti}$ and $\mathrm{Nb}_{3} \mathrm{Sn}$ coils to He II," Phys. Rev. Spec. Top. Accel. Beams, vol. 11, p. 082401, 2008.

[5] D. Tommasini and D. Richter, "A new cable insulation scheme improving heat transfer in $\mathrm{Nb}$-Ti superconducting accelerator magnets,' in Proc. 11th European Particle Accelerator Conference, Genoa, 2008, p. WEPD027.

[6] P. Fessia and P. P. Granieri et al., "Electrical and mechanical performance of an enhanced cable insulation scheme for superconducting magnets," IEEE Trans. Appl. Supercond., submitted for publication.

[7] O. Bruning et al., LHC Design Report vol. 1 CERN, Geneva, Switzerland, 2004, pp. 157, 170.

[8] C. Meuris et al., "Heat transfer in electrical insulation of LHC cables cooled with superfluid helium," Cryogenics, vol. 39, pp. 921-931, 1999.

[9] B. Baudouy et al., "He II heat transfer through superconducting cables electrical insulation," Cryogenics, vol. 40, pp. 127-136, 2000.

[10] D. Richter et al., "Evaluation of the transfer of heat from the coil of the LHC dipole magnet to helium II," IEEE Trans. Appl. Supercond., vol. 17, no. 2, pp. 1263-1268, 2007.

[11] N. Kimura et al., "Heat transfer characteristics of Rutherford-type superconducting cables in pressurized He II," IEEE Trans. Appl. Supercond., vol. 9, no. 2, pp. 1097-1100, 1999.

[12] P. P. Granieri, Mechanical Measurements at Ambient Temperature of an Enhanced Cable Insulation Scheme for the Superconducting Magnets of the LHC Luminosity Upgrade TE/MSC internal note to be published, CERN. Geneva, Switzerland, 2009. 